\title{
Predicting long-term disease control in transplant-ineligible patients with multiple myeloma: impact of an MGUS-like signature
}

\author{
Paula Rodríguez-Otero', María Victoria Mateos², Joaquín Martínez-López³ , Miguel-Teodoro Hernández \\ Enrique M. Ocio ${ }^{2}$, Laura Rosiñol ${ }^{5}$, Rafael Martínez ${ }^{6}$, Ana-lsabel Teruel${ }^{7}$, Norma C. Gutiérrez ${ }^{2}$, Joan Bargay ${ }^{8}$, \\ Enrique Bengoechea ${ }^{9}$, Yolanda González ${ }^{10}$, Jaime Pérez de Oteyza ${ }^{11}$, Mercedes Gironella ${ }^{12}$, Jorge M. Nuñez-Córdoba ${ }^{13}$, \\ Cristina Encinas $^{14}$, Jesús Martín ${ }^{15}$, Carmen Cabrera ${ }^{16}$, Luis Palomera ${ }^{17}$, Felipe de Arriba ${ }^{18}$, María Teresa Cedena ${ }^{3}$, \\ Noemí Puig ${ }^{2}$, Albert Oriol ${ }^{19}$, Bruno Paiva ${ }^{1}$, Joan Bladé ${ }^{6}$, Juan José Lahuerta ${ }^{4}$ and Jesús F. San Miguel ${ }^{1}$
}

\begin{abstract}
Disease control at 5 years would be a desirable endpoint for elderly multiple myeloma (MM) patients, but biomarkers predicting this are not defined. Therefore, to gain further insights in this endpoint, a population of 498 newly diagnosed transplant-ineligible patients enrolled in two Spanish trials (GEM2005MAS65 and GEM2010MAS65), has been analyzed. Among the 435 patients included in this post-hoc study, 18.6\% remained alive and progression free after 5 years of treatment initiation. In these patients, overall survival (OS) rate at 10 years was $60.8 \%$ as compared with $11.8 \%$ for those progressing within the first 5 years. Hemoglobin $(\mathrm{Hb}) \geq 12 \mathrm{~g} / \mathrm{dl}(\mathrm{OR} 2.74, p=0.001)$ and MGUS-like profile (OR 4.18, $p=0.005$ ) were the two baseline variables associated with long-term disease-free survival. Upon including depth of response (and MRD), Hb $\geq 12 \mathrm{~g} / \mathrm{dl}$ (OR 2.27) and MGUS-like signature (OR 7.48) retained their predictive value along with MRD negativity (OR 5.18). This study shows that despite the use of novel agents, the probability of disease control at 5 years is still restricted to a small fraction (18.6\%) of elderly MM patients. Since this endpoint is associated with higher rates of OS, this study provides important information about diagnostic and posttreatment biomarkers helpful in predicting the likelihood of disease control at 5 years.
\end{abstract}

\section{Introduction}

Treatment of multiple myeloma (MM) has significantly improved in the last decade but the outcome remain significantly poorer in elderly patients ${ }^{1}$. Thus, while approximately $34-60 \%$ of transplant-eligible cases remain progression free at 5 years $^{2-4}$ and this has resulted in

\footnotetext{
Correspondence: Jesús F. San Miguel (sanmiguel@unav.es)

${ }^{1}$ Clínica Universidad de Navarra, CIMA, IDISNA, CIBERONC, Pamplona, Spain

${ }^{2}$ Complejo Asistencial Universitario de Salamanca, Instituto de Investigación Biomédica de Salamanca, Salamanca, Spain

Full list of author information is available at the end of the article.

These authors contributed equally: Paula Rodríguez-Otero, María Victoria

Mateos
}

unprecedent rates $(10-40 \%)$ of 10 -year overall survival $(\mathrm{OS})^{2-4}$; the percentage of elderly patients reaching disease control at 5 years and the characteristics of such patients is less well established. Moreover, in the nontransplant-eligible population, definition of long-term disease control could be different, since patients that are free from relapse beyond 5 years, could eventually die for reasons other than progressive disease. Thus, achieving disease control beyond 5 years would be a most relevant endpoint, since elderly patients usually have fewer options for subsequent lines of therapy, and a prolonged first

\section{(c) The Author(s) 2019}

(c) (i) Open Access This article is licensed under a Creative Commons Attribution 4.0 International License, which permits use, sharing, adaptation, distribution and reproduction cc) in any medium or format, as long as you give appropriate credit to the original author(s) and the source, provide a link to the Creative Commons license, and indicate if changes were made. The images or other third party material in this article are included in the article's Creative Commons license, unless indicated otherwise in a credit line to the material. If material is not included in the article's Creative Commons license and your intended use is not permitted by statutory regulation or exceeds the permitted use, you will need to obtain permission directly from the copyright holder. To view a copy of this license, visit http://creativecommons.org/licenses/by/4.0/. 
response will translate not only into a longer survival but also in improved quality of life.

Several studies have searched for relevant prognostic factors associated with prolonged survival in elderly MM. Thus, in addition to a fit status, according to geriatric assessment $^{5}$, and absence of high-risk cytogenetic abnormalities (HR CAs) ${ }^{6}$, both the International Staging System (ISS) and the Revised-ISS (R-ISS) ${ }^{7,8}$ clearly identified that ISS 1 and R-ISS 1 patients enjoy a significantly longer progression-free survival (PFS) and OS. Additional conventional factors such as normal hemoglobin $(\mathrm{Hb})$, creatinine, and beta-2-microglobulin (B2M) levels, age below 80 years and normal lactate dehydrogenase (LDH) levels, as well as, low percentage of S-phase bone marrow (BM) plasma cells (PCs), have been identified as predictors for longer PFS and $\mathrm{OS}^{9-11}$. Nevertheless, none of these studies have specifically focused on the identification of the characteristics of the patients' cohort that achieve a long-term disease control (PFS $>5$ years).

Depth of response is also associated with prolonged survival in elderly patients. Thus, complete response (CR) defined by conventional criteria translates into longer PFS and $\mathrm{OS}^{12-14}$. More recently, it has been shown that the achievement of sustained minimal residual disease negativity clearly predict for a longer PFS and OS in non-transplant candidate patients ${ }^{15}$. However, there is a small subset of MM patients that despite persistence of the disease enjoy long-term disease control, underlying the need of new tools to identify these long-term survivors.

Interestingly, it has been reported that patients with a previous diagnosis of monoclonal gammopathy of unknown significance (MGUS) or smoldering MM (SMM) have a more prolonged survival ${ }^{16}$. However, although it is well known that all MM evolve from a prior MGUS, in most patients presenting with active disease no information on a previous MGUS-status is available. Noteworthy, both the Arkansas ${ }^{17}$ and the Spanish groups ${ }^{18}$ have reported, by using gene expression profiling (GEP) and multiparameter flow cytometry (MFC) techniques, respectively, that an MGUS signature, associated with low-risk features and superior survival, can be identified in a fraction of patients with active MM.

Here, we evaluated a large series of 498 transplantineligible MM patients enrolled in two consecutive Spanish clinical trials, treated with first-generation novel agent-based combinations and with mature follow-up. Our aims were to (i) determine the percentage of elderly patients with disease control at 5 years after treatment initiation, (ii) analyze whether this translates into a substantial benefit in OS, and (iii) define an optimal set of biomarkers to estimate the odds of achieving disease control at 5 years.

\section{Patients and methods \\ Study design}

Four hundred ninety-eight newly diagnosed elderly and symptomatic MM patients (aged 65 years or older) were included in two prospective Spanish trials: GEM2005MAS65 and GEM2010MAS65 ${ }^{19-22}$. Patients with a follow-up shorter than 5 years $(n=22)$ or who died without progression $(n=41)$ were censored from the analysis and, accordingly, 435 patients were evaluated in this post-hoc study. All patients were diagnosed according to the IMWG criteria ${ }^{23}$. Data cut-off was 20 February 2018. All patients provided written informed consent before screening. Data were monitored by an external contract research organization and centrally re-assessed. Median follow-up was 92.6 months (range: 0.3-132.9 months) and 61.7 (range: 1.6-81.1 months) for the GEM2005MAS65 and the GEM2010MAS65 studies, respectively. The endpoint of this study was defined as time to progression at 5 years or longer since diagnosis, in patients with a follow-up of at least 5 years.

The GEM05MAS65 trial included 259 patients randomized to receive upfront induction treatment with either bortezomib, melphalan, and prednisone (VMP), or bortezomib, thalidomide, and prednisone $(\mathrm{VTP})^{16}$. VMP induction therapy consisted of six cycles: one cycle of intravenous bortezomib given twice per week for 6 weeks $\left(1.3 \mathrm{mg} / \mathrm{m}^{2}\right.$ on days $1,4,8,11,22,25,29$, and 32$)$, plus oral melphalan $9 \mathrm{mg} / \mathrm{m}^{2}$ and prednisone $60 \mathrm{mg} / \mathrm{m}^{2}$ on days $1-4$, followed by five cycles of bortezomib once per week for 5 weeks $\left(1.3 \mathrm{mg} / \mathrm{m}^{2}\right.$ on days $1,8,15$, and 22) plus the same doses of melphalan and prednisone. VTP induction therapy consisted of the same schedule of bortezomib and prednisone plus oral, continuous, thalidomide at a dose of $100 \mathrm{mg}$ per day instead of melphalan. Patients from each arm completing the six induction cycles were then randomly assigned to maintenance therapy with either bortezomib and thalidomide (VT) or bortezomib and prednisone (VP). Maintenance consisted of one conventional cycle of bortezomib $\left(1.3 \mathrm{mg} / \mathrm{m}^{2}\right.$ on days $1,4,8$, and 11 ) every 3 months, plus either oral prednisone $(50 \mathrm{mg}$ every other day) or oral thalidomide (50 $\mathrm{mg}$ per day), for up to 3 years.

In the GEM010MAS65, 239 patients were randomly assigned 1:1 to receive both VMP and lenalidomide in combination with low-dose dexamethasone (Rd) in a sequential or alternating manner, for 18 cycles $^{17}$. VMP therapy comprised nine cycles: one 6-week cycle of IV bortezomib using a conventional twice-weekly schedule $\left(1.3 \mathrm{mg} / \mathrm{m}^{2}\right.$ on days $1,4,8,11,22,25,29$, and 32$)$, plus oral melphalan $\left(9 \mathrm{mg} / \mathrm{m}^{2}\right)$ and oral prednisone $(60 \mathrm{mg} /$ $\mathrm{m}^{2}$ ) on days 1-4, followed by eight 4-week cycles of onceweekly IV bortezomib ( $1.3 \mathrm{mg} / \mathrm{m}^{2}$ on days $1,8,15$, and 22$)$ plus the same doses of melphalan and prednisone. $\mathrm{Rd}$ treatment consisted of nine cycles of oral lenalidomide 
$25 \mathrm{mg}$ daily on days $1-21$ of each 28 -day cycle plus oral dexamethasone $40 \mathrm{mg}$ weekly, on days $1,8,15$, and 22 .

The following characteristics, documented at diagnosis, were analyzed: age, gender, $\mathrm{Hb}$, serum creatinine levels, B2M, albumin, PC infiltration, $\mathrm{LDH}$, type of monoclonal component, presence of urine light chains, extramedullary disease, clinical staging according to the ISS and R-ISS, plus chromosomal abnormalities defining high-risk patients: $t(4 ; 14)$ and/or $t(14 ; 16)$ and/or $\operatorname{del}(17 \mathrm{p})$.

In addition, an immunophenotypic algorithm to determine if patients had MGUS-like vs MM-like profiles was used by comparing the relative frequency of BM PCs plus the percentage of clonal and normal PCs within the whole $\mathrm{BM}$ PC compartment for each patient, plotted against a database containing information on the same three parameters from a total of 1774 patients, including 497 MGUS and 1277 newly diagnosed MM enrolled in the GEM2000 $(N=486)$ plus GEM2005MENOS65 $(N=330)$ protocols for transplant-eligible cases, and the GEM2005MENOS65 $(N=239)$ plus GEM2010MAS65 $(N=222)$ protocols for transplant-ineligible patients. Based on those three parameters and the automatic population separator (APS) representation of the Infinicyt software (Cytognos, Salamanca, Spain), two reference groups corresponding to MGUS and MM emerged; each patient falling into the MGUS reference being classified as MGUS-like (Supplementary Figure 1$)^{18}$.

Fluorescence in situ hybridization (FISH) on CD138purified PCs and immunophenotyping were performed centrally at diagnosis following standard procedures ${ }^{6}$. Responses were evaluated according to the European Group for Blood and Marrow Transplantation ${ }^{24}$ and IMWG $^{25}$ criteria.

\section{Statistical analysis}

The endpoint of this study was defined as time to progression at 5 years or longer since diagnosis, in patients with a follow-up of at least 5 years.

The baseline characteristics of the patients are presented as mean and standard deviation (SD) for continuous variables and as proportions for categorical variables. Univariate analysis was performed using the chi-squared or Fisher's exact test, as appropriate, for independent variables. Multivariate analysis was performed using a forward-stepwise model. Variable selection to be tested in the multivariate analysis was carried out using an automated selection method (forward-stepwise). Two significance levels were specified in the forward-stepwise procedure: $10 \%$ for predictor addition to the model, and $20 \%$ for predictor removal from the model. A first model including all the baseline variables was performed and a second model including depth of response based on conventional and minimal residual disease evaluation was conducted using the same criteria.
In both models, the R-ISS was included instead of ISS, $\mathrm{LDH}$, and HR CAs in order to avoid redundancy. A landmark analysis at 6 and 12 months has been performed including all patients. Crude survival analysis was assessed using log-rank test. Kaplan-Meier survival analysis was carried out for each group category. All statistical analyses were carried out using Stata 12.1.

\section{Results}

The median PFS for the whole population included in the GEM05MAS65 and GEM2010MAS65 $(n=498)$ was 31.4 month (95\% confidence inerval (CI), 28.28-34.36), being 28.3 months (95\% CI, 24.8-32.9) and 34.3 months (95\% CI, 29.6-39.1) in each trial, respectively. The median OS was 57.3 months (95\% CI, 49.1-65.4), being 51.6 months $(95 \% \mathrm{CI}, 42.9-61.1)$ and 68.2 months $(95 \%$ CI, 49.1-NR) for patients included in the GEM05MAS65 and GEM2010MAS65 trials, respectively.

Four hundred thirty-five newly diagnosed transplantineligible MM patients were analyzed in this post-hoc study. Eighty-one out of 435 patients (18.6\%) were alive and without disease progression during the first 5 years of initiating treatment and this group of patients has been identified as the long-term disease-free survivor group. The remaining 354 patients have progressed in the first 5 years after the diagnosis. The proportion of patients alive at 10 years in the long-term disease-free survivor group was $60.8 \%(95 \% \mathrm{CI}, \quad 35.4 \%-78.8 \%) \quad$ as compared with $11.8 \%$ (95\% CI, $5.9 \%-19.8 \%)(p<0.001)$, respectively (Fig. 1).

Results of the univariate analyses are summarized in Table 1. Baseline variables significantly associated with long-term PFS were, ISS 1 (35\% vs $22.3 \%$; $p=0.036$ ), RISS 1 (30\% vs $16.6 \%, p=0.002), \mathrm{Hb} \geq 12 \mathrm{~g} / \mathrm{dl}(34.6 \%$ vs $17.8 \%, p=0.001)$, normal LDH $(97.5 \%$ vs $87.6 \%, \mathrm{p}=$ 0.009 ), and absence of HR CAs (91\% vs $80.9 \%, p=0.04$ ).

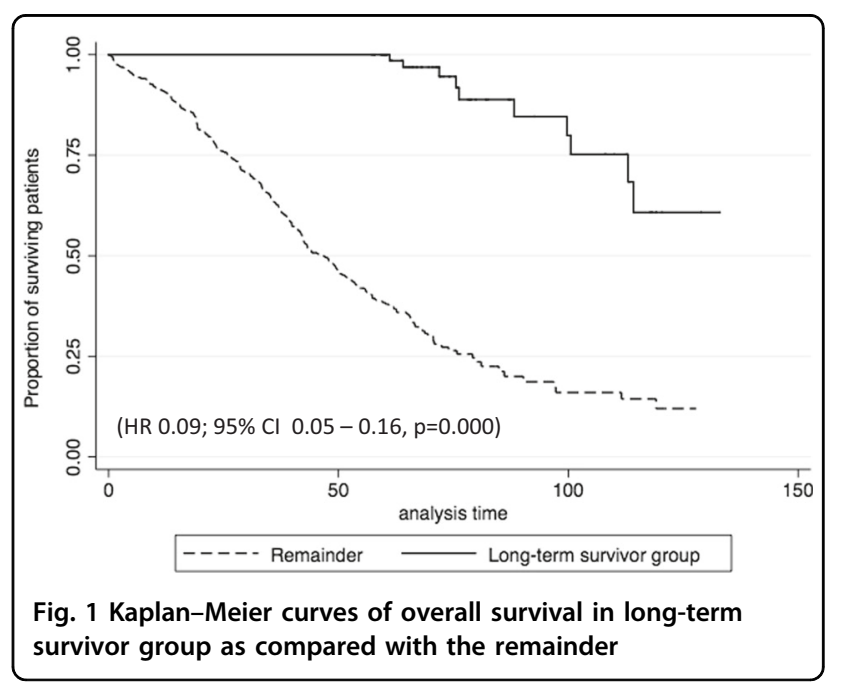


Table 1 Distribution of the main characteristic between the long-term survival group and the remainder population (univariate analysis)

\begin{tabular}{|c|c|c|c|}
\hline Variable & $\begin{array}{l}\text { Long-term survival, } \\
n(\%)\end{array}$ & Remainder, $n$ (\%) & $p$-Value \\
\hline Age & $n=81$ & $n=354$ & $p=$ n.s. \\
\hline$<75$ years & $56(69.1)$ & $224(63.2)$ & \\
\hline Gender & $n=81$ & $n=354$ & $p=$ n.s. \\
\hline Male & $46(56.8)$ & $178(50.3)$ & \\
\hline Protocol & $n=81$ & $n=354$ & $p=$ n.s. \\
\hline GEM05MAS65 & $44(54.3)$ & $206(58.2)$ & \\
\hline GEM2010MAS65 & $37(45.7)$ & $148(41.8)$ & \\
\hline Durie stage & $n=81$ & $n=352$ & $p=$ n.s. \\
\hline I & $12(14.8)$ & $29(8.2)$ & \\
\hline$\|$ & $42(51.8)$ & $168(47.8)$ & \\
\hline III & $27(33.3)$ & $155(44)$ & \\
\hline ISS & $n=80$ & $n=350$ & $p=0.036$ \\
\hline ISS 1 & $28(35)$ & $78(22.3)$ & \\
\hline ISS 2 & $33(41.2)$ & $151(43.1)$ & \\
\hline ISS 3 & $19(23.8)$ & $121(34.6)$ & \\
\hline R-ISS & $n=80$ & $n=350$ & $p=0.002$ \\
\hline R-ISS 1 & $24(30)$ & $58(16.6)$ & \\
\hline R-ISS 2 & $55(68.8)$ & $256(73.1)$ & \\
\hline R-ISS 3 & $1(1.2)$ & $36(10.3)$ & \\
\hline Hemoglobin (g/dl) & $n=81$ & $n=354$ & $p=0.001$ \\
\hline $\mathrm{Hb} \geq 12 \mathrm{~g} / \mathrm{dl}$ & $28(34.6)$ & $63(17.8)$ & \\
\hline Creatinine $(\mathrm{mg} / \mathrm{dl})$ & $n=81$ & $n=354$ & $p=$ n.s. \\
\hline Creat $<1.1$ & $54(66.7)$ & $242(68.4)$ & \\
\hline LDH & $n=81$ & $n=348$ & $p=0.009$ \\
\hline Normal & 79 (97.5) & $305(87.6)$ & \\
\hline Cytogenetics & $n=75$ & $n=335$ & $p=0.04$ \\
\hline Standard risk & $68(91)$ & $271(80.9)$ & \\
\hline Response & $n=81$ & $n=327$ & $p=0.001$ \\
\hline $\mathrm{CR}(\mathrm{s} C \mathrm{R}+\mathrm{CR} \mathrm{IF}-)$ & $47(58)$ & $109(33.3)$ & \\
\hline VGPR & $27(33.3)$ & $161(49.2)$ & \\
\hline$P R$ & $4(4.9)$ & $45(13.8)$ & \\
\hline SD & $3(3.7)$ & $11(3.4)$ & \\
\hline PD & $0(0)$ & $1(0.3)$ & \\
\hline BM signature & $n=77$ & $N=323$ & $p<0.001$ \\
\hline MGUS-like profile & $12(15.6)$ & $12(3.7)$ & \\
\hline MM-like profile & $68(84.4)$ & $311(96.3)$ & \\
\hline MRD & $n=81$ & $n=354$ & $p<0.001$ \\
\hline Negative & $35(41.9)$ & $35(9.9)$ & \\
\hline
\end{tabular}

n.s. not statistically significant, $M R D$ minimal residual disease, $C R$ complete response, $s C R$ stringent complete response, $C R$ IF- complete response with negative immunofixation, VGPR very good partial response, $P R$ partial response, $S D$ stable disease, $P D$ progressive disease, $\mathrm{Hb}$ hemoglobin, Creat creatinine, ISS International Staging System, R-ISS revised International Staging System, LDH lactate deshydrogenase

Moreover, patients in the long-term disease-free survivor group presented more frequently an MGUS-like profile ( $15.6 \%$ vs $3.7 \%, p<0.001)$. In addition, quality of response measure by both the proportion of complete remissions (CRs) (58\% vs $33.3 \%, p=0.001)$, and MRD-negative cases $(41.9 \%$ vs $9.9 \%, p<0.001)$ were also higher in the longterm PFS group.
Table 2a Multivariate analysis including baseline variables

\begin{tabular}{llll}
\hline & Odds ratio & 95\% Cl & $\boldsymbol{p}$-Value \\
\hline MGUS-like profile & 4.18 & $1.55-11.26$ & 0.005 \\
$\mathrm{Hb} \geq 12 \mathrm{~g} / \mathrm{dl}$ & 2.74 & $1.53-4.89$ & 0.001 \\
\hline $\begin{array}{l}\text { MGUS monoclonal gammopathy of unknown significance, } \mathrm{Hb} \text { hemoglobin, 95\% } \\
\text { Cl 95\% confidence interval }\end{array}$
\end{tabular}

Table 2b Multivariate analysis including depth of response measured by complete response and MRD negativity rates

\begin{tabular}{llll}
\hline & Odds ratio & $\mathbf{9 5 \% ~ C l}$ & $\boldsymbol{p}$-Value \\
\hline MGUS-like profile & 7.48 & $2.46-22.74$ & 0.000 \\
$\mathrm{Hb} \geq 12 \mathrm{~g} / \mathrm{dl}$ & 2.27 & $1.22-4.22$ & 0.009 \\
MRD negativity & 5.18 & $2.77-9.69$ & 0.000 \\
\hline MGUS monoclonal gammopathy of unknown significance, $\mathrm{Hb}$ hemoglobin, MRD \\
negativity minimal residual disease negativity detected by flow cytometry
\end{tabular}

The multivariate logistic regression analysis showed that the probability of long-term disease control was associated with two independent baseline factors (Table 2a): the presence of an $\mathrm{Hb} \geq 12 \mathrm{~g} / \mathrm{dl}$ (OR 2.74; 95\% CI, 1.53-4.89, $p=0.001$ ), and the presence of an MGUS-like profile in the BM PCs (OR 4.18; 95\% CI, 1.5-11.3, $p=$ 0.005). When incorporating depth of response and patients' MRD status into the model, three variables were found to be independently associated with the probability of 5-year PFS (Table 2b): the presence of an MGUS-like profile (OR 7.48; 95\% CI, 2.46-22.74, $p<0.001$ ); the MRD negativity (OR 5.18; 95\% CI, 2.77-9.69, $p<0.001$ ), and the $\mathrm{Hb} \geq 12 \mathrm{~g} / \mathrm{dl}$ (OR 2.27; 95\% CI, 1.22-4.22, $p=0.009$ ). Moreover, after performing a landmark analysis at 6 and 12 months including all patients $(n=498)$, the three variables significantly associated with long-term survival remain the same $(\mathrm{Hb}>12$, MGUS-like profile and MRD negativity).

Focusing on the 24 patients with an MGUS-like signature, $50 \%$ of these patients displayed a long-term disease-free survival, as compared with $17.3 \%$ of the remaining MM patients. The proportion of patients alive at 10 years with an MGUS-like profile was $40.5 \%$ (95\% CI, 9.4-70.7) as compared with $20.3 \%$ (95\% CI, 12.7-29.2) in the MM-like population $(p=0.07)$. Similarly, for PFS, the proportion of patients alive and without disease progression at 10 years in the MGUS-like group was $40.2 \%$ (95\% CI, 20.5-59.1) as compared with 5.7\% (95\% CI, 2.8-10.1) among the MM-like patients $(p=0.001)$. Disease response has been evaluated in 21 out of 24 patients with MGUS-like profile and $90 \%$ of the patients achieved a favorable response (10 CR and 9 VGPR). No differences in 
outcome were observed between VGPR and CR cases ( $p$ value for OS 0.78) among the MGUS-like profile patients. Interestingly, as suggested by the multivariate analysis, the impact of the MGUS-like signature on long-term survival is independent on the MRD status, since only $16.7 \%$ of these patients achieved MRD negativity, as compared with $46.15 \%$ patients in the long-term survival group with a MM-like profile $(p=0.057)$.

\section{Discussion}

In the transplant setting, it has been clearly shown that improving the median PFS beyond 5 years resulted in median OS of $8-12$ years $^{2-4}$. However, it is not so well established if disease control at 5 years in elderly MM has a similar impact in OS. Indeed, in this setting it may be even more relevant, since many elderly patients will only receive 2-3 lines of therapy, and accordingly, a prolonged initial response could translate both in a prolonged survival, similar to that of elderly healthy individuals, and an improved quality of life. The definition of long-term disease control used in the present study (PFS beyond 5 years) is quite challenging since it is equivalent to the median OS reported in the VISTA ${ }^{26}$ and FIRST trials ${ }^{27}$ based on VMP (OS: 56 months) or continuous Lenalidomide-dexamethasone (OS: 59 months) treatments, respectively. Upon considering VRD as a new standard of care, the median PFS reported for patients above the age of 65 ( 35 months) is clearly inferior to the 5 years here proposed ${ }^{28}$. Taken together, it should be assumed that despite substantial improvement in the treatment of MM, the proportion of elderly patients achieving long-term disease control (i.e.: $>5$ years PFS) is limited and factors identifying this population upfront are lacking.

In our series, only $18.6 \%$ out of 435 elderly patients entered in this category, and $61 \%$ of them achieved an OS of more than 10 years, accordingly they will reach a life expectancy similar to the normal population. As expected, this patients' cohort is characterized by the presence of low-risk clinical features (R-ISS 1, normal Hb, and LDH). The ISS, particularly the revised version (R-ISS), that include the cytogenetic information, is a powerful tool in discriminating risk subgroups, but their predictive value for 5-year disease control is not established. By contrast, the emergence of normal $\mathrm{Hb}$ levels as a strong predictor of long-term disease control was surprising and could reflect a lower $\mathrm{BM}$ infiltration and potentially a better tolerance to treatment. But the most relevant biomarker for predicting long-term disease control upfront was the presence of an MGUS-like signature defined by automated MFC and characterized by a low number of bone BM PCs together with a relative preservation in the percentage of normal vs clonal PCs within the whole BM PC compartment. The favorable outcome of patients with an
MGUS-like profile was first shown by the Arkansas group using a gene expression signature based on 52 genes $^{17}$. From 214 patients with MM, 27\% were found to have an MGUS-like signature, and it was associated with low-risk clinical and molecular features and superior survival. Moreover, the MGUS-like signature was also seen in PCs from 15 of 20 patients surviving $>10$ years after autotransplantation. By using MFC, Paiva et al. ${ }^{18}$ had shown that 59 out of 689 transplant candidate MM patients (8\%) displayed an MGUS-like profile. Despite achieving similar CR rates after high-dose therapy vs other MM patients, MGUS-like cases had very long time to progression and OS $(\sim 60 \%$ at 10 years; $p<0.001)$. Although, MGUS-like signature does not necessarily drive a long time to progression, our data indicate that this biomarker can contribute to identify a subset of symptomatic MM patients with an excellent outcome, independently of the depth of response. Overall, these data could also imply that treating patients before massive disease dissemination and niche occupancy in BM confers favorable prognosis.

Although the depth of response is not predictable at the time of diagnosis, it was shown to be a significant predictive marker of long-term disease control when measured with conventional methods (CR) or, particularly, by MFC (MRD negativity). Noteworthy, our study highlights that the prognostic impact of a MGUS-like signature, is even more relevant than to achieve MRD negativity for predicting long-term disease control (HR of 7.09 and 4.87, respectively). Although MRD is becoming a most valuable treatment endpoint and a surrogate marker for survival, the fact that MGUS-like and MRD are independent confirms that for some patients the achievement of an MRD-negative status is not imperative for attaining longterm disease control.

In summary, we identified that the combination of three biomarkers (normal Hb, MGUS-like signature, and MRD negativity) can help to define elderly $M M$ patients achieving long-term disease control. Although these findings will be validated in our next GEM2017FIT trial for elderly newly diagnosed MM patients, our results show that the presence of a MGUS-like signature in the $\mathrm{BM}$ at diagnoses is the most powerful predictor for longterm disease-free survival, becoming an important prognostic biomarker.

\footnotetext{
Acknowledgements

This study was supported by the Centro de Investigación Biomédica en RedÁrea de Oncología_del Instituto de Salud Carlos III (CIBERONC; CB16/12/ 00369 and CB16/12/00377), Instituto de Salud Carlos III/Subdirección General de Investigación Sanitaria (FIS no. PS09/01897/01370, PI12/01761, PI12/02311, PI13/01469, PI14/01867, G03/136, CD13/00340), Asociación Española Contra el Cáncer (GCB120981SAN) and the European Research Council (ERC) 2015 Starting Grant (MYELOMANEXT).

\section{Author details}

${ }^{1}$ Clínica Universidad de Navarra, CIMA, IDISNA, CIBERONC, Pamplona, Spain. ${ }^{2}$ Complejo Asistencial Universitario de Salamanca, Instituto de Investigación
} 
Biomédica de Salamanca, Salamanca, Spain. ${ }^{3}$ Hospital Universitario 12 de Octubre, Instituto de Investigación 12 de Octubre, CIBERONC, Madrid, Spain. ${ }^{4}$ Hospital Universitario de Canarias, Santa Cruz de Tenerife, Spain. ${ }^{5}$ Hospital Clinic I Provincial, Institud'Investigacions Biomèdiques August Pi i Sunyer (IDIBAPS), Barcelona, Spain. ${ }^{6}$ Hospital Clínico San Carlos, Madrid, Spain. ${ }^{7}$ Hospital Clínico de Valencia, Valencia, Spain. ${ }^{8}$ Hospital Son Llatzer, Palma de Mallorca, Spain. ${ }^{9}$ Hospital de Donostia, San Sebastian, Spain. ${ }^{10}$ Institut d'Oncologia Dr. Josep Trueta, Girona, Spain. "11 Hospital de Madrid Sanchinarro, Universidad CEU San Pablo, Madrid, Spain. ${ }^{12}$ Hospital Vall d'Hebron, Barcelona, Spain. ${ }^{13}$ Research Support Service, Central Clinical Trials Unit, University Clinic of Navarra, Pamplona, Spain. ${ }^{14}$ Hospital General Universitario Gregorio Marañón, Instituto de Investigación Sanitaria Gregorio Marañón (liSGM), Madrid, Spain. ${ }^{15}$ Hospital General Virgen del Rocío, Sevilla, Spain. ${ }^{16}$ Hospital San Pedro de Alcántara, Cáceres, Spain. ${ }^{17}$ Hospital Clínico Universitario Lozano Blesa, Zaragoza, Spain. ${ }^{18}$ Servicio de Hematología y Oncología Médica, Hospital Universitario Morales Meseguer, IMIB-Arrixaca, Universidad de Murcia, Murcia, Spain. ${ }^{19}$ Insitut Català d'Oncologia, Institut Josep Carreras, Hospital German Trias i Pujol, Badalona, Barcelona, Spain

\section{Conflict of interest}

P.R.-O.: has served on the speaker's bureau and as a member of advisory boards for Janssen, Celgene, BMS, and Takeda. M.V.M.: has declared consultancy for: Janssen, Celgene, Takeda, and Amgen. E.M.O.: has declared consultancy for or honoraria from: Mundipharma, Celgene, Amgen, Novartis, Takeda, AbbVie, BMS, and Janssen. B.P.: has declared grants from Celgene, EngMab, Sanofi, and Takeda, and received honoraria for lectures and advisory boards from Amgen, BMS, Celgene, Janssen, Takeda, Sanofi, and Novartis. J.F.S.M.: received honoraria as part of Advisory boards from Celgene, Novartis, Millenium, Janssen, Amgen, MSD, Sanofi, and BMS. A.O. has served as a member of advisory boards for Janssen and Celgene. J.B.: received honoraria for lectures and advisory boards from Celgene and Janssen and grant support from Janssen. L.R.: received honoraria from Janssen and Celgene. The remaining authors declare that they have no conflict of interest.

\section{Publisher's note}

Springer Nature remains neutral with regard to jurisdictional claims in published maps and institutional affiliations.

Supplementary information accompanies this paper at (https://doi.org/ 10.1038/s41408-019-0176-X).

Received: 13 September 2018 Revised: 28 December 2018 Accepted: 14 January 2019

Published online: 18 March 2019

\section{References}

1. Fonseca, R. et al. Trends in overall survival and costs of multiple myeloma, 2000-2014. Leukemia 31, 1915-1921 (2017).

2. Barlogie, B. et al. Long-term follow-up of autotransplantation trials for multiple myeloma: update of protocols conducted by the intergroupe francophone du myelome, southwest oncology group, and university of arkansas for medical sciences. J. Clin. Oncol. 28, 1209-1214 (2010).

3. Cavo, M. et al. International Myeloma Working Group consensus approach to the treatment of multiple myeloma patients who are candidates for autologous stem cell transplantation. Blood 117, 6063 (2011).

4. Tacchetti P. et al. Abstract triplet bortezomib- and immunomodulator-based therapy before and afer double ASCT improves overall survival of newly diagnosed MM ptients: final analysis of Phase 3 GIMEMA-MMY-3006 study. Abstract \#S105. 23rd Congress of the European Hematology Association; 14-17 June 2018; Stockholm, SE.

5. Palumbo, A. et al. Geriatric assessment predicts survival and toxicities in elderly myeloma patients: an International Myeloma Working Group report. Blood 125, 2068-2074 (2015).

6. Gutierrez, N. C. et al. Prognostic and biological implications of genetic abnormalities in multiple myeloma undergoing autologous stem cell transplantation: $\mathrm{t}(4 ; 14)$ is the most relevant adverse prognostic factor, whereas RB deletion as a unique abnormality is not associated with adverse prognosis. Leukemia 21, 143-150 (2007).

7. Palumbo, A. et al. Revised International Staging System for multiple myeloma: a report from International Myeloma Working Group. J. Clin. Oncol. 33, 2863-2869 (2015).

8. Greipp, P. R. et al. International Staging System for multiple myeloma. J. Clin. Oncol. 23, 3412-3420 (2005).

9. Ludwig, $\mathrm{H}$. et al. Myeloma in patients younger than age 50 years presents with more favorable features and shows better survival: an analysis of 10549 patients from the International Myeloma Working Group. Blood 111, 4039-4047 (2008)

10. Stella-Holowiecka, B. et al. Beta-2-microglobulin level predicts outcome following autologous hematopoietic stem cell transplantation in patients with multiple myeloma. Transplant. Proc. 39, 2893-2897 (2007).

11. San Miguel, J. F. et al. A new staging system for multiple myeloma based on the number of S-phase plasma cells. Blood 85, 448-455 (1995).

12. Harousseau, J. L. et al. Superior outcomes associated with complete response in newly diagnosed multiple myeloma patients treated with nonintensive therapy: analysis of the phase 3 VISTA study of bortezomib plus melphalanprednisone versus melphalan-prednisone. Blood 116, 3743-3750 (2010).

13. Gay, F. et al. Complete response correlates with long-term progression-free and overall survival in elderly myeloma treated with novel agents: analysis of 1175 patients. Blood 117, 3025-3031 (2011).

14. Kyle, R. A. et al. Complete response in multiple myeloma: clinical trial E9486, an Eastern Cooperative Oncology Group study not involving stem cell transplantation. Cancer 106, 1958-1966 (2006).

15. Lahuerta, J. J. et al. Depth of response in multiple myeloma: a pooled analysis of three PETHEMAVGEM clinical trials. J. Clin. Oncol. 35, 2900-2910 (2017).

16. Sigurdardottir, E. E. et al. The role of diagnosis and clinical follow-up of monoclonal gammopathy of undetermined significance on survival in multiple myeloma. JAMA Oncol. 1, 168-174 (2015).

17. Zhan, F. et al. Gene-expression signature of benign monoclonal gammopathy evident in multiple myeloma is linked to good prognosis. Blood 109, 1692 (2007).

18. Paiva, B. et al. A multiparameter flow cytometry immunophenotypic algorithm for the identification of newly diagnosed symptomatic myeloma with an MGUS-like signature and long-term disease control. Leukemia 27, 2056-2061 (2013).

19. Mateos, M. V. et al. Sequential vs alternating administration of VMP and Rd in elderly patients with newly diagnosed MM. Blood 127, 420-425 (2016).

20. Mateos, M. V. et al. Bortezomib, melphalan, and prednisone versus bortezomib, thalidomide, and prednisone as induction therapy followed by maintenance treatment with bortezomib and thalidomide versus bortezomib and prednisone in elderly patients with untreated multiple myeloma: a randomised trial. Lancet Oncol. 11, 934-941 (2010).

21. Mateos, M. V. et al. Maintenance therapy with bortezomib plus thalidomide or bortezomib plus prednisone in elderly multiple myeloma patients included in the GEM2005MAS65 trial. Blood 120, 2581-2588 (2012).

22. Mateos, M. V. et al. GEM2005 trial update comparing VMPNTP as induction in elderly multiple myeloma patients: do we still need alkylators? Blood 124, 1887-1893 (2014).

23. International Myeloma Working Group. Criteria for the classification of monoclonal gammopathies, multiple myeloma and related disorders: a report of the International Myeloma Working Group. Br. J. Haematol. 121, 749-757 (2003).

24. Bladé, J. et al. Criteria for evaluating disease response and progression in patients with multiple myeloma treated by high-dose therapy and haemopoietic stem cell transplantation. Br. J. Haematol. 102, 1115-1123 (1998).

25. Durie, B. G. et al. International uniform response criteria for multiple myeloma. Leukemia 20, 1467-1473 (2006).

26. San Miguel, J. F. et al. Persistent overall survival benefit and no increased risk of second malignancies with bortezomib-melphalan-prednisone versus melphalan-prednisone in patients with previously untreated multiple myeloma. J. Clin. Oncol. 31, 448-455 (2013).

27. Facon T. et al. Final analysis of overall survival from the first trial. Blood Abstracts: 58th Annual Meeting Abstracts. 1 Dec 2016;128.

28. Durie, B. G. et al. Bortezomib with lenalidomide and dexamethasone versus lenalidomide and dexamethasone alone in patients with newly diagnosed myeloma without intent for immediate autologous stem-cell transplant (SWOG S0777): a randomised, open-label, phase 3 trial. Lancet 389, 519-527 (2017). 